\title{
The Brown-York mass of black holes in Warped Anti-de Sitter space
}

\author{
Gaston Giribet ${ }^{1, *}$ and Andrés Goya ${ }^{1, \text {, }}$ \\ ${ }^{1}$ Physics Department, University of Buenos Aires FCEN-UBA, and IFIBA-CONICET, \\ Ciudad Universitaria, Pabellón I, 1428, Buenos Aires, Argentina.
}

\begin{abstract}
We give a direct computation of the mass of black holes in Warped Anti-de Sitter space (WAdS) in terms of the Brown-York stress-tensor at the boundary. This permits to explore to what extent the holographic renormalization techniques can be applied to such type of deformation of AdS. We show that, despite some components of the boundary stress-tensor diverge and resist to be regularized by the introduction of local counterterms, the precise combination that gives the quasilocal energy density yields a finite integral. The result turns out to be in agreement with previous computations of the black hole mass obtained with different approaches. This is seen to happen both in the case of Topologically Massive Gravity and of the so-called New Massive Gravity. Here, we focus our attention on the latter. We observe that, despite other conserved charges diverge in the near boundary limit, the finite part in the large radius expansion captures the physically relevant contribution. We compute the black hole angular momentum in this way and we obtain a result that is in perfect agreement with previous calculations.
\end{abstract}

PACS numbers: $11.25 . \mathrm{Tq}, 11.10 . \mathrm{Kk}$

\section{INTRODUCTION}

The idea of extending AdS/CFT correspondence to Warped AdS spaces (WAdS) has been originally proposed in Ref. [1], and it was further studied in Refs. [2-11]. This represents one of the most appealing attempts to generalize holography to non-AdS spaces, and this is because WAdS spaces appear in several contexts. For instance, WAdS spaces provide gravity duals for condensed matter systems with Schrödinger symmetry [12, 13], they are closely related to the geometry of rotating black holes [14, 15], and they also appear in relation to many other interesting subjects 16 18. Asymptotically $\mathrm{WAdS}_{3}$ spaces turn out to be exact solutions of String Theory [19, 20] as well as of other models of threedimensional gravity, including Higher-Spin Gravity [21], Topologically Massive Gravity (TMG) 22, 23], and New Massive Gravity (NMG) [24]. Here, we will be concerned with the latter: We will consider asymptotically $\mathrm{WAdS}_{3}$ black holes in NMG. For such solutions, we will give a direct computation of the mass in terms of the BrownYork stress-tensor [25] in the boundary of the space. We do this to explore to what extent the holographic renormalization techniques can be applied to such a deformation of AdS. Whether or not the Brown-York tensor can be defined at the boundary of $\mathrm{WAdS}_{3}$ space is a question that has been raised, for instance, in Ref. [5]. Here, we will show that, despite some components of the Brown-York stress-tensor diverge in the near boundary limit and resist to be regularized by the introduction of local counterterms, the integral of the precise combination that gives the definition of the quasilocal energy as a conserved charge yields a finite integral. The result we

\footnotetext{
* Electronic address: gaston-at-df.uba.ar
}

$\dagger^{\dagger}$ Electronic address: af.goya-at-df.uba.ar obtain happens to be in agreement with computations of the black hole mass obtained by different methods [22, 27, 28]. Finiteness of the conserved charge computed in this way follows from cancellations that occur near the boundary. In contrast to the mass, in the case of the angular momentum the charge associated to it can not be regularized by the introduction of local boundary counterterms. However, the finite part in the near boundary expansion happens to capture the physically relevant information, and it is shown to exactly reproduce the black hole angular momentum.

The paper is organized as follows: In Section II, we briefly review the theory of New Massive Gravity introduced in Ref. 29]. In Section III, we discuss the geometry of Warped Anti-de Sitter space and black holes that asymptote to it. In Section IV, we study boundary terms and the Brown-York stress-tensor they induce. We consider the near boundary limit of this stress-tensor and use it in Section V to calculate the mass of the Warped Antide Sitter black holes. That is, we compute the BrownYork quasilocal energy in the limit that the boundary tends to spatial infinity. The result we obtain is in agreement with previous computations. We also discuss the analogous computation in the case of the gravitational Chern-Simons term being added.

\section{NEW MASSIVE GRAVITY}

Let us begin by reviewing New Massive Gravity theory 29]. The action of the theory consists of the sum of three different contributions, namely

$$
S=S_{\mathrm{EH}}+S_{\mathrm{NMG}}+S_{\mathrm{B}},
$$

where the first term is the Einstein-Hilbert action with cosmological constant,

$$
S_{\mathrm{EH}}=\frac{1}{16 \pi G} \int_{\Sigma} d^{3} x \sqrt{-g}(R-2 \Lambda),
$$


and the second term contains contributions of higher order,

$$
\begin{aligned}
S_{\mathrm{NMG}}= & \frac{1}{16 \pi G} \int_{\Sigma} d^{3} x \sqrt{-g}\left(f^{\mu \nu} G_{\mu \nu}-\right. \\
& \left.\frac{1}{4} m^{2}\left(f_{\mu \nu} f^{\mu \nu}-f^{2}\right)\right)
\end{aligned}
$$

where $G_{\mu \nu}$ is the Einstein tensor $G_{\mu \nu}=R_{\mu \nu}-\frac{1}{2} R g_{\mu \nu}$, and field $f_{\mu \nu}$ is a rank-two auxiliary field which, after being integrated, gives

$$
f_{\mu \nu}=\frac{2}{m^{2}}\left(R_{\mu \nu}-\frac{1}{4} R g_{\mu \nu}\right) .
$$

The third term in (1), $\mathrm{S}_{B}$, is a boundary action needed for the variational principle to be defined in a specific way. We will discuss the boundary terms later.

By reinserting (4) back in (3) the higher-curvature terms take the form

$$
S_{\mathrm{NMG}}=\frac{1}{16 \pi G m^{2}} \int_{\Sigma} d^{3} x \sqrt{-g}\left(R_{\mu \nu} R^{\mu \nu}-\frac{3}{8} R^{2}\right)
$$

which is the form of the action presented in 29].

The equations of motion derived from action (11) read

$$
16 \pi G \frac{\delta S}{\delta g^{\mu \nu}}=G_{\mu \nu}+\Lambda g_{\mu \nu}+\frac{1}{2 m^{2}} K_{\mu \nu}=0 .
$$

which, apart from the Einstein tensor $G_{\mu \nu}$, involve the tensor

$$
\begin{aligned}
K_{\mu \nu}= & 2 \square R_{\mu \nu}-\frac{1}{2} \nabla_{\mu} \nabla_{\nu} R-\frac{1}{2} \square R g_{\mu \nu}+4 R_{\mu \alpha \nu \beta} R^{\alpha \beta}- \\
& \frac{3}{2} R R_{\mu \nu}-R_{\alpha \beta} R^{\alpha \beta} g_{\mu \nu}+\frac{3}{8} R^{2} g_{\mu \nu}
\end{aligned}
$$

The precise combination of the square-curvature terms in (5), $g^{\mu \nu} K_{\mu \nu}=R_{\mu \nu} R^{\mu \nu}-(3 / 8) R^{2}$, is such that the trace of the equations of motion (6) does not involve the mode $\square R$. This is one of the reasons why NMG is free of ghosts - for instance - about flat space.

Equations of motion (6) are solved by all solutions of General Relativity, provided an adequate renormalization of the effective cosmological constant. The theory also admits solutions that are not Einstein spaces; these have $K_{\mu \nu} \neq 0$. Probably the simplest solutions of this sort are $\mathrm{WAdS}_{3}$ spaces.

\section{WARPED ANTI-DE SITTER}

\section{A. WAdS $_{3}$ space}

WAdS $_{3}$ spaces are squashed or stretched deformations of $\mathrm{AdS}_{3}$ [14]. Such a deformation is obtained by first writing $\mathrm{AdS}_{3}$ as a Hopf fibration of $\mathbb{R}$ over $\mathrm{AdS}_{2}$ and then multiplying the fiber by constant warp factor $K$. More precisely, one first considers the metric of $\mathrm{AdS}_{3}$ written in coordinates

$$
d s^{2}=\frac{l^{2}}{4}\left(-\cosh ^{2} x d \tau^{2}+d x^{2}+(d y+\sinh x d \tau)^{2}\right)
$$

and then deforms it as follows

$$
d s^{2}=\frac{l_{K}^{2}}{4}\left(-\cosh ^{2} x d \tau^{2}+d x^{2}+K(d y+\sinh x d \tau)^{2}\right)
$$

where $x, y, \tau \in \mathbb{R}$, and $K \in \mathbb{R}$. It is usual to parameterize the deformation by a positive constant $\nu$ defined by $K=$ $4 \nu^{2} /\left(\nu^{2}+3\right)$, such that $\nu=1$ corresponds to undeformed - unwarped- $\mathrm{AdS}_{3}$. Through the deformation, the $\mathrm{AdS}_{3}$ radius $l$ gets also rescaled as $l^{2} \rightarrow l_{K}^{2}=4 l^{2} /\left(\nu^{2}+3\right)$. Spaces (9) with $\nu^{2}>1$ describe stretched $\mathrm{AdS}_{3}$ spaces, while those with $\nu^{2}<1$ describe squashed deformations of it. Through a double Wick rotation $x, \tau \rightarrow i x, i \tau$ one goes from the spacelike $\mathrm{WAdS}_{3}$ metric (9) to a timelike analog of it. Here we will be involved with spacelike stretched $\mathrm{WAdS}_{3}$ spaces.

\section{B. $\mathbf{W A d S}_{3} / \mathrm{CFT}_{2}$}

The warping deformation breaks the $S L(2, \mathbb{R}) \times$ $S L(2, \mathbb{R})$ isometry group of $\mathrm{AdS}_{3}$ space down to $S L(2, \mathbb{R}) \times U(1)$. As a consequence, also the asymptotic isometry group, which in the case of $\mathrm{AdS}_{3}$ coincides with the two-dimensional local conformal group, gets altered. It has been recently understood that the asymptotic group of $\mathrm{WAdS}_{3}$ turns out to be generated by the semi-direct product of one copy of Virasoro algebra and an affine extension of $u(1)$ algebra with non-vanishing central extension; see [2-4, 8, 9]. That is, the asymptotic isometry group in $\mathrm{WAdS}_{3}$ spaces certainly differs from the two-dimensional conformal group; nevertheless, it has been shown in [11] that, under certain circumstances, the symmetry results powerful enough to constrain the dual theory and extract relevant information from it. The holographic description of $\mathrm{WAdS}_{3}$ black hole thermodynamics carried out in [11] is a notable realization of this idea.

Motivated by the similarities between asymptotically $\mathrm{WAdS}_{3}$ and asymptotically $\mathrm{AdS}_{3}$ spaces, the authors of [1] proposed the idea of extending AdS/CFT to the former case. The conjecture is that quantum gravity in asymptotically $\mathrm{WAdS}_{3}$ space would be dual to a twodimensional theory which exhibits partial conformal symmetry, it being symmetric under right - but not left dilations. The main motivation we have to study the holographic renormalization techniques in this context comes from trying to determine to what extent what we know about holography can be applied with no major modification to WAdS spaces as well. 


\section{WAdS $_{3}$ black holes}

One of the most attractive properties of WAdS space is that it admits black holes that asymptote to it and, on the other hand, are given by discrete quotients of $\mathrm{WAdS}_{3}$ itself. This is analogous to what happens with the Bañados-Teiltelboim-Zanelli (BTZ) black hole [30], which is locally equivalent to $\mathrm{AdS}_{3}$. The existence of $\mathrm{WAdS}_{3}$ black holes is very interesting since, if thought of within the context of a $\mathrm{WAdS}_{3} / \mathrm{CFT}_{2}$ correspondence, it gives raise the hope to investigate black hole physics in a totally new setup.

The metric of $\mathrm{WAdS}_{3}$ black holes is given by

$$
\begin{aligned}
d s^{2}= & d t^{2}+\left(2 \nu r-\sqrt{\left(\nu^{2}+3\right) r_{+} r_{-}}\right) d t d \varphi+ \\
& l^{2}\left(\left(\nu^{2}+3\right)\left(r-r_{+}\right)\left(r-r_{-}\right)\right)^{-1} d r^{2}+ \\
& \frac{r}{4}\left(3\left(\nu^{2}-1\right) r+\left(\nu^{2}+3\right)\left(r_{+}+r_{-}\right)-\right. \\
& \left.4 \nu \sqrt{r_{+} r_{-}\left(\nu^{2}+3\right)}\right) d \varphi^{2}
\end{aligned}
$$

where $t \in \mathbb{R}$, the angular coordinate $\varphi \in[0,2 \pi)$, it being identified as $\varphi \sim \varphi+2 \pi$, and $r \in \mathbb{R}_{\geq 0} . r_{+}$and $r_{-}$are two integration constants that, for $r_{+} \geq r_{-} \geq 0$, represent the location of the outer and inner horizons of the black hole. Solutions (10) asymptote spacelike stretched $\mathrm{WAdS}_{3}$ at large $r$. Metric (10) can also be written in the ADM like form

$$
d s^{2}=-N_{t}^{2} d t^{2}+\rho^{2}\left(d \varphi+N^{\varphi} d t\right)^{2}+\frac{l^{2} d r^{2}}{4 \rho^{2} N_{t}^{2}},
$$

with

$$
\begin{aligned}
\rho^{2}= & \frac{r}{4}\left(3\left(\nu^{2}-1\right) r+\left(\nu^{2}+3\right)\left(r_{+}+r_{-}\right)-\right. \\
& \left.4 \nu \sqrt{r_{+} r_{-}\left(\nu^{2}+3\right)}\right), \\
N_{t}^{2}= & \frac{\left(\nu^{2}+3\right)\left(r-r_{+}\right)\left(r-r_{-}\right)}{4 \rho^{2}}, \\
N^{\varphi}= & \frac{2 \nu r-\sqrt{\left(\nu^{2}+3\right) r_{+} r_{-}}}{2 \rho^{2}} .
\end{aligned}
$$

As mentioned, $\mathrm{WAdS}_{3}$ black holes are specific identifications of the $\mathrm{WAdS}_{3}$ space [1]. That is, black hole geometry (10) is constructed as a quotient of $\mathrm{WAdS}_{3}$ space by a discrete subgroup of $S L(2, \mathbb{R}) \times U(1)$, identifying points of the original manifold along a direction $\partial_{\varphi}=\pi l\left(J_{2} / \beta_{\mathrm{L}}-\bar{J}_{2} / \beta_{\mathrm{R}}\right)$, with $J_{2} \in S L(2, \mathbb{R})$ and $\bar{J}_{2} \in U(1)$, and $\beta_{\mathrm{L}, \mathrm{R}} \in \mathbb{R}$. This allows to define the leftand right-temperature as the inverse of the periods $\beta_{\mathrm{R}, \mathrm{L}}$; namely

$$
\begin{aligned}
& T_{\mathrm{L}}=\beta_{\mathrm{L}}^{-1}=\frac{\left(\nu^{2}+3\right)}{8 \pi l^{2}}\left(r_{+}+r_{-}-\frac{1}{\nu} \sqrt{\left(\nu^{2}+3\right) r_{+} r_{-}}\right), \\
& T_{\mathrm{R}}=\beta_{\mathrm{R}}^{-1}=\frac{\left(\nu^{2}+3\right)}{8 \pi l^{2}}\left(r_{+}-r_{-}\right) .
\end{aligned}
$$

Because of being locally equivalent to $\mathrm{WAdS}_{3}$ space (9), and despite having a richer causal structure, the local geometry of black holes (10) is remarkably simple. In particular, the curvature scalars result to be independent of the integration constants $r_{ \pm}$. Moreover, all the curvature invariants turn out to be constant, given only in terms of parameters $\nu$ and $l$; for instance,

$$
\begin{gathered}
R=-\frac{6}{l^{2}}, \quad R_{\mu \nu} R^{\mu \nu}=\frac{6}{l^{4}}\left(3-2 \nu^{2}+\nu^{4}\right), \\
R_{\mu \nu} R_{\rho}^{\nu} R^{\rho \mu}=-\frac{6}{l^{6}}\left(9-9 \nu^{2}+3 \nu^{4}+\nu^{6}\right) .
\end{gathered}
$$

As we will see, this geometric simplicity of $\mathrm{WAdS}_{3}$ black holes is, paradoxically, one of the aspects that make difficult to deal with them.

\section{NMG WAdS 3 black holes}

It has been shown in 24] that $\mathrm{WAdS}_{3}$ black holes (10) solve the equations of motion of NMG if the parameters satisfy the relations

$$
m^{2}=-\frac{\left(20 \nu^{2}-3\right)}{2 l^{2}}, \Lambda=-\frac{m^{2}\left(9-48 \nu^{2}+4 \nu^{2}\right)}{\left(9-120 \nu^{2}+400 \nu^{4}\right)} .
$$

The same type of solution for the case of NMG theory coupled to TMG was studied in Ref. [27].

Entropy of $\mathrm{WAdS}_{3}$ black hole in NMG can be evaluated by means of Wald formula [31], yielding

$$
S=\frac{8 \pi \nu^{3}}{\left(20 \nu^{2}-3\right) G}\left(r_{+}-\frac{1}{2 \nu} \sqrt{\left(\nu^{2}+3\right) r_{+} r_{-}}\right) .
$$

Remarkably, the entropy results proportional to $T_{\mathrm{L}}+$ $T_{\mathrm{R}}$, which means that it admits to be written in the Cardy like form

$$
S=\frac{\pi^{2} l}{3} c\left(T_{\mathrm{L}}+T_{\mathrm{R}}\right)
$$

with $c$ being independent of $r_{ \pm}$. Then, one may identify the central charge of the dual theory to be

$$
c=\frac{96 \nu^{3} l}{\left(20 \nu^{4}+57 \nu^{2}-9\right) G} .
$$

Notice that, in the limit $\nu \rightarrow 1$, central charge (18) tends to its $\mathrm{AdS}_{3}$ value $c=24 l /(17 G)$; recall that in NMG the Brown-Henneaux central charge $3 l /(2 G)$ gets multiplied by a factor $1+1 /\left(2 m^{2} l^{2}\right)$, and, according (15), $\nu=1$ corresponds to $m^{2} l^{2}=-17 / 2$.

\section{BROWN-YORK STRESS-TENSOR}

\section{A. ADM decomposition}

Now, let us analyze the definition of the Brown-York tensor in NMG. This has been originally studied in Ref. 
[32]. To define the stress-tensor, it is convenient to write the metric in its ADM decomposition for the radial coordinate, $r$, namely

$$
d s^{2}=N^{2} d r^{2}+\gamma_{i j}\left(d x^{i}+N^{i} d r\right)\left(d x^{j}+N^{j} d r\right),
$$

where $N^{2}$ is the radial lapse function, and $\gamma_{i j}$ is the two-dimensional metric on the constant- $r$ surfaces. The Latin indices $i, j=0,1$, refer to the coordinates on the constant- $r$ surfaces, while the Greek indices are $\mu, \nu=$ $0,1,2$, and include the radial direction $r$ as well. In the case of asymptotically $\mathrm{AdS}_{3}$ spaces, one knows how to restrict the $r$-dependence of $\gamma_{i j}$ as it comes from the Fefferman-Graham expansion [33], which in threedimensions results consistent with the Brown-Henneaux asymptotic boundary conditions 34]. For $\mathrm{WAdS}_{3}$ the asymptotic boundary conditions were studied in Refs. [2[4, 8, 9] for the case of TMG; in particular, it has been shown in [4] that the theory admits more than one set of consistent boundary conditions, all of them being defined in a way that $\mathrm{WAdS}_{3}$ black hole solutions (10) are gathered. We assume such kind of asymptotic behavior. More precisely, we consider perturbation of the $r_{+}=r_{-}=0$ configuration (10) of the form

$$
\begin{aligned}
d s^{2}= & d t^{2}+2 \nu r d t d \varphi+\frac{l^{2} d r^{2}}{r^{2}\left(\nu^{2}+3\right)}+ \\
& \frac{3 r}{4}\left(\nu^{2}-1\right) d \varphi^{2}+h_{\mu \nu} d x^{\mu} d x^{\nu}
\end{aligned}
$$

gathering metrics with falling-off conditions

$$
\begin{aligned}
& h_{r r} \simeq \mathcal{O}\left(r^{-3}\right), \quad h_{\varphi \varphi} \simeq \mathcal{O}(r), \\
& h_{t \varphi} \simeq \mathcal{O}(1), \quad h_{t t} \simeq \mathcal{O}\left(r^{-3}\right) .
\end{aligned}
$$

\section{B. Boundary terms}

Boundary terms $S_{\mathrm{B}}$ are introduced in (11) for the variational principle to be defined in such a way that both the metric $g_{\mu \nu}$ and the auxiliary field $f_{\mu \nu}$ are fixed on the boundary $\partial \Sigma$. With this prescription, the boundary action $S_{\mathrm{B}}$ reads

$$
S_{\mathrm{B}}=-\frac{1}{8 \pi G} \int_{\partial \Sigma} d^{2} x \sqrt{-\gamma}\left(K+\frac{1}{2} \hat{f}^{i j}\left(K_{i j}-\gamma_{i j} K\right)\right) .
$$

Here, $\gamma_{i j}$ is the metric induced on $\partial \Sigma$ and $K_{i j}$ is the extrinsic curvature, with $K=\gamma^{i j} K_{i j}$. On the other hand, $\hat{f}^{i j}$ in (21) comes from decomposing the contravariant field $f^{\mu \nu}$ as

$$
f^{\mu \nu}=\left(\begin{array}{cc}
f^{i j} & h^{j} \\
h^{i} & s
\end{array}\right)
$$

and defining

$$
\hat{f}^{i j} \equiv f^{i j}+2 h^{(i} N^{j)}+s N^{i} N^{j}, \quad \hat{f} \equiv \gamma_{i j} \hat{f}^{i j} .
$$

The first term in (21) corresponds to the GibbonsHawking term. The other two terms come from the higher-curvature terms of NMG. Notice that in (21) the field $\hat{f}^{i j}$ couples to the Israel tensor $K_{i j}-\gamma_{i j} K$ in the same manner as the field $f^{\mu \nu}$ couples to the Einstein tensor in the bulk action (3).

Then, the Brown-York stress-tensor can be obtained by varying action (1) with respect to the metric $\gamma^{i j}$; namely

$$
T_{i j}=\frac{2}{\sqrt{-\gamma}} \frac{\delta S}{\delta \gamma^{i j}}{ }_{\mid r=\mathrm{const}} .
$$

This yields two distinct contributions, $T^{i j}=T_{\mathrm{EH}}^{i j}+$ $T_{\mathrm{NMG}}^{i j}$. First, we have the Israel term

$$
T_{\mathrm{EH}}^{i j}=\frac{1}{8 \pi G}\left(K^{i j}-K \gamma^{i j}\right)
$$

and, secondly, we have the contribution coming from the higher-curvature terms 32.

$$
\begin{aligned}
T_{\mathrm{NMG}}^{i j}= & -\frac{1}{8 \pi G}\left(\frac{1}{2} \hat{f} K^{i j}+\nabla^{(i} \hat{h}^{j)}-\frac{1}{2} \nabla_{r} \hat{f}^{i j}+K_{k}^{(i} \hat{f}^{j) k}-\right. \\
& \left.\frac{1}{2} \hat{s} K^{i j}-\gamma^{i j}\left(\nabla_{k} \hat{h}^{k}-\frac{1}{2} \hat{s} K+\frac{1}{2} \hat{f} K-\frac{1}{2} \nabla_{r} \hat{f}\right)\right),
\end{aligned}
$$

where $\hat{h^{i}}=N\left(h^{i}+s N^{i} N^{j}\right), \hat{s}=N^{2} s$, and where the covariant $r$-derivative $\nabla_{r}$ is defined as follows

$$
\begin{aligned}
\nabla_{r} \hat{f}^{i j} & =\frac{1}{N}\left(\partial_{r} \hat{f}^{i j}-N^{k} \partial_{k} \hat{f}^{i j}+2 \hat{f}^{k(i} \partial_{k} N^{j)}\right), \\
\nabla_{r} \hat{f} & =\frac{1}{N}\left(\partial_{r} \hat{f}-N^{k} \partial_{k} \hat{f}\right) .
\end{aligned}
$$

\section{Counterterms}

The next step towards the definition of the boundary stress-tensor is adding counterterms to regularize (22) in the limit $r \rightarrow \infty$. In asymptotically $\mathrm{AdS}_{3}$ space this is achieved by the holographic renormalization recipe, which amounts to add boundary terms that only involve intrinsic boundary quantities. Here, such terms would be of the form

$$
S_{\mathrm{C}}=\frac{1}{8 \pi G} \int_{\partial \Sigma} d^{2} x \sqrt{-\gamma}\left(a_{0}+a_{1} \hat{f}+a_{2} \hat{f}^{2}+b_{2} \hat{f}_{i j} \hat{f}^{i j}+\ldots\right)
$$

The ellipses stand for higher-order intrinsic terms. From the boundary viewpoint these terms are thought of as counterterms in the dual theory; meaning that the renormalized boundary stress-tensor is defined by taking the $r \rightarrow \infty$ limit of the improved stress-tensor

$$
T_{i j} \rightarrow T_{i j}^{*}=T_{i j}+\frac{2}{\sqrt{-\gamma}} \frac{\delta S_{\mathrm{C}}}{\delta \gamma^{i j}}
$$

The choice of counterterms (23), namely the choice of coefficients $a_{i}, b_{i}$, is partially determined by demanding 
the action to be finite. Regarding this point, it is worthwhile mentioning a peculiarity of $\mathrm{WAdS}_{3}$ space, which is the fact that $\mathrm{WAdS}_{3}$ space does not admit a real Euclidean section. Therefore, one has to specify precisely what does requiring finiteness in the action actually mean in this context. We will circumvent the problem by saying that here we are dealing with stationary solutions, so we will demand the Lorentzian action integrated over a finite time interval to be finite. This is achieved by choosing

$$
a_{0}=-\frac{8 \nu^{2} \sqrt{\nu^{2}+3}}{\left(20 \nu^{2}-3\right) l} .
$$

Nevertheless, one may ask whether (25) is the only possible choice. It turns out that the answer is in the affirmative: In contrast to what happens with other solutions of massive gravity, like the ones found in Refs. [35] and [36], whose boundary stress-tensors can be regularized by introducing additional counterterms, here the geometrical simplicity of the $\mathrm{WAdS}_{3}$ spaces happens to play against us: For $\mathrm{WAdS}_{3}$ we have

$$
\begin{gathered}
\hat{f}=-\frac{2 \nu^{2}}{m^{2} l^{2}}, \quad \hat{f}_{i j} \hat{f}^{i j}=\frac{2}{m^{4} l^{4}}\left(9-18 \nu^{2}+10 \nu^{4}\right), \\
\hat{f}_{i j} \hat{f}_{k}^{j} \hat{f}^{k i}=-\frac{2 \nu^{2}}{m^{6} l^{6}}\left(27-54 \nu^{2}+28 \nu^{4}\right), \ldots
\end{gathered}
$$

and, therefore, there are no many options as all the terms are constant. Thus, the frugal menu of counterterms we have at hand has only one independent option, say (25). This is precisely what we meant when we said that the geometric simplicity of $\mathrm{WAdS}_{3}$ black holes is one of the aspects that make difficult to deal with them.

Notice that counterterm (25) is consistent with the fact that $\mathrm{WAdS}_{3}$ black holes reduce to BTZ black hole (in a rotating frame) when $\nu=1$. For $\nu=1$ we have $a_{0}=$ $-16 /(17 l)$, which is the expected value for the case in which there is no warping and $\mathrm{WAdS}_{3}$ space reduces to $\mathrm{AdS}_{3}$. Recall that $m^{2}=-\left(20 \nu^{2}-3\right) /\left(2 l^{2}\right)$, so that for $\nu=1$ we have $2 l^{2} m^{2}=-17$; on the other hand, in NMG the counterterm needed to regularize the boundary stress-tensor in $\mathrm{AdS}_{3}$ is a boundary cosmological term with coefficient $a_{0}=-\left(1+2 m^{2} l^{2}\right) /\left(2 m^{2} l^{3}\right)=-16 /(17 l)$.

\section{CONSERVED CHARGES}

\section{A. Quasilocal energy}

Once the stress-tensor has been improved by adding to it the boundary contributions $S_{\mathrm{C}}$ that render the Lagrangian finite, one can define the conserved charges as follows 25$]$

$$
Q[\xi]=\int d s u^{i} T_{i j}^{*} \xi^{j}
$$

where $d s$ is the line element of the constant- $t$ surfaces at the boundary, $u$ is a unit vector orthogonal to the constant- $t$ surfaces, and $\xi$ is the Killing vector that generates the isometry in $\partial \Sigma$ to which the charge is associated. In the case of the mass, the components of this vector are $\xi^{i}=N_{t} u^{i}$, where the lapse function $N_{(r)}^{t}$ in (11). This defines the energy density; see 25, 37] for discussions. From (11) we see that the line element $d s$ in the case we are interested in is simply $d s=\rho d \varphi$.

However, before going further, let us express a concern about the finiteness of (26). This is because the fact that counterterm (23) achieves to make the action finite in the way we discussed it, does not necessarily imply that the stress-tensor is finite as well. In fact, it can be explicitly verified that the inclusion of counterterm (23) with (25) in the case of $\mathrm{WAdS}_{3}$ black holes does not suffice to make all the components of $T_{i j}^{*}$ finite. Nevertheless, it turns out that, despite the divergences in the improved stress-tensor, the charge (26) defined with $\xi=N_{t} u$ at the boundary $r=\infty$ results finite. It gives

$$
M=\frac{\nu^{2}\left(\nu^{2}+3\right)}{2\left(20 \nu^{2}-3\right) l G}\left(r_{+}+r_{-}-\frac{1}{\nu} \sqrt{\left(\nu^{2}+3\right) r_{+} r_{-}}\right) .
$$

This agrees with the result obtained in [24, 27, 28] up to a factor $1 / 2$. The comparison with the mass computed in 24] is discussed in Appendix C of Ref. [27]. Finiteness of (27) follows from cancellations that take place in the near boundary limit. This can be verified by the large $r$ expansion of the $T_{i j}$ components

$$
\begin{aligned}
T_{t t} & \simeq t_{t t}^{(0)}+t_{t t}^{(-1)} r^{-1}+t_{t t}^{(-2)} r^{-2}+\mathcal{O}\left(r^{-3}\right), \\
T_{t \varphi} & \simeq t_{t \varphi}^{(1)} r+t_{t \varphi}^{(0)}+t_{t \varphi}^{(-1)} r^{-1}+\mathcal{O}\left(r^{-2}\right),
\end{aligned}
$$

where $t_{i j}^{(n)}$ are constant coefficients, and the large $r$ expansion of the unit vector components

$$
\begin{aligned}
u^{t} & \simeq u_{(0)}^{t}+u_{(-1)}^{t} r^{-1}+\mathcal{O}\left(r^{-2}\right), \\
u^{\varphi} & \simeq u_{(-1)}^{\varphi} r^{-1}+\mathcal{O}\left(r^{-2}\right) .
\end{aligned}
$$

Coefficient $u_{(-1)}^{t}$ results proportional to (27).

As mentioned in Ref. [26] in a similar context, mass formula (27) is cumbersome enough for not to doubt about its calculation by means of (24)-(26) actually makes sense. Nevertheless, to convince ourselves about it, let us revise the same type of calculation for TMG and see that it also works when the gravitational ChernSimons term is included.

\section{B. Gravitational Chern-Simons term}

Certainly, $\mathrm{WAdS}_{3}$ spaces were first obtained as exact solutions to the equations of motion of TMG [22, 23]. $\mathrm{WAdS}_{3}$ space and $\mathrm{WAdS}_{3}$ black holes are solutions to TMG if the coupling of the Chern-Simons term,

$$
S_{\mathrm{CS}}=\frac{1}{32 \pi G \mu} \int_{\Sigma} d^{3} x \varepsilon^{\mu \nu \rho} \Gamma_{\mu \alpha}^{\eta}\left(\partial_{\nu} \Gamma_{\rho \eta}^{\alpha}+\frac{2}{3} \Gamma_{\nu \beta}^{\alpha} \Gamma_{\rho \eta}^{\beta}\right),
$$


and the parameters $\nu, l$ satisfy the relation $\nu=\mu l / 3$. For $\mathrm{WAdS}_{3}$ black holes of TMG, it had already been observed in [26] that the computation of the mass using the Brown-York tensor in the boundary yielded the result

$$
M=\frac{\left(\nu^{2}+3\right)}{48 l G}\left(r_{+}+r_{-}-\frac{1}{\nu} \sqrt{\left(\nu^{2}+3\right) r_{+} r_{-}}\right),
$$

which, again, is in notable agreement with other calculations using different methods, cf. 11. In the case of TMG, the large $r$ limit of $Q[\xi]$ is regularized by introducing a boundary cosmological term with coefficient

$$
a_{0}=-\frac{\sqrt{\nu^{2}+3}}{2 l}
$$

which also tends to the $\mathrm{AdS}_{3}$ value, $-1 / l$, in the limit $\nu=1$. Therefore, the computation of the $\mathrm{WAdS}_{3}$ black hole mass in terms of the boundary stress-tensor is seen to work in different scenarios.

\section{Angular momentum}

Now, let us go back to NMG. In contrast to the computation of the mass $M=Q\left[N_{t} u\right]$, charge $Q\left[\partial_{\varphi}\right]$, which is associated to the $\mathrm{WAdS}_{3}$ black hole angular momentum, does not yield a finite result in the limit $r \rightarrow \infty$. In fact, boundary terms (23) do not suffice to regularize the divergences appearing in the charge $Q\left[\partial_{\varphi}\right]=\int d s u^{i} T_{i \varphi}^{*}$. This is simply expressed by the fact that $u_{\varphi}=0$. Nevertheless, the finite part of the large $r$ expansion of $Q\left[\partial_{\varphi}\right]$ happens to capture the physically relevant information. This can be seen by looking at the stress-tensor expansion

$$
T_{\varphi \varphi} \simeq t_{\varphi \varphi}^{(2)} r^{2}+t_{\varphi \varphi}^{(1)} r+t_{\varphi \varphi}^{(0)}+\mathcal{O}\left(r^{-1}\right),
$$

which results in an expansion of the form

$$
Q\left[\partial_{\varphi}\right] \simeq J_{(2)} r^{2}+J_{(1)} r+J_{(0)}+\mathcal{O}\left(r^{-1}\right),
$$

where the coefficient $J_{(2)}$ depends only on $\nu$, while coefficient $J_{(1)}$ depends both on $\nu$ and $r_{ \pm}$; namely

$$
\begin{aligned}
J_{(2)}= & \frac{9}{8} \frac{\nu\left(\nu^{2}-1\right)^{2}}{\left(20 \nu^{2}-3\right) l} \\
J_{(1)}= & \frac{3}{8} \frac{\nu\left(\nu^{2}-1\right)^{2}}{\left(20 \nu^{2}-3\right) l}\left(\left(\nu^{2}+3\right)\left(r_{+}+r_{-}\right)-\right. \\
& 4 \nu \sqrt{\left.\left(\nu^{2}+3\right) r_{+} r_{-}\right)} .
\end{aligned}
$$

From this expansion it is not hard to verify that, in contrast to the case of the mass, for $\nu^{2} \neq 1$ the introduction of only local counterterms (23) does not produce contributions to cancel the divergences in $Q\left[\partial_{\varphi}\right]$. However, remarkably enough, the finite part $J_{(0)}$ gives the correct result for the black hole angular momentum; that is,

$$
\begin{aligned}
J_{(0)}= & \frac{\nu\left(\nu^{2}+3\right)}{4\left(20 \nu^{2}-3\right) G l}\left(\left(5 \nu^{2}+3\right) r_{+} r_{-}-\right. \\
& \left.2 \nu \sqrt{\left(\nu^{2}+3\right) r_{+} r_{-}}\left(r_{+}+r_{-}\right)\right) .
\end{aligned}
$$

To see that (30) actually reproduces the correct result one may resort to the computation done in Ref. [24], where the Abbott-Deser-Tekin [39, 40] method to compute conserved charges was used to obtain the $\mathrm{WAdS}_{3}$ black hole angular momentum. The result obtained in 24] reads

$$
\tilde{J}=\frac{\zeta^{3} \eta^{2}}{4 G m^{2} l^{2}}\left(\left(1-\eta^{2}\right) \omega^{2}-\frac{\rho_{0}^{2}}{\left(1-\eta^{2}\right)}\right)
$$

where $\zeta=2 \nu, \eta=-\sqrt{\nu^{2}+3} /(2 \nu), \omega=\left(r_{+}+r_{-}+\right.$ $\left.2 \eta \sqrt{r_{+} r_{-}}\right) /\left(2-2 \eta^{2}\right)$, and $\rho_{0}^{2}=\left(r_{+}-r_{-}\right)^{2} / 4$. Then, after translating (31) to our notation, one verifies that (30) is proportional to (31), namely $\tilde{J}=J_{(0)} \zeta^{2}\left(1-\eta^{2}\right) / 4$, and the proportionality factor is precisely the (square of the) one that relates the angular coordinates $\phi$ used in Ref. [24] and our angular coordinate $\varphi$; more precisely, we have $\phi=\varphi \sqrt{\zeta^{2}\left(1-\eta^{2}\right)} / 2$. This proportionality factor $\zeta^{2}\left(1-\eta^{2}\right) / 4$ is also explained in Appendix $\mathrm{C}$ of Ref. [27]; see equation (C.15) therein. In conclusion, the finite part of charge $Q\left[\partial_{\varphi}\right]$ captures the physically relevant contribution and gives the correct value of the black hole angular momentum (30). The question remains as to how to understand the failure in regularizing $Q\left[\partial_{\varphi}\right]$ as a consequence of the abstruse asymptotic structure of $\mathrm{WAdS}_{3}$ spaces.

This work has been supported by UBA, CONICET, and ANPCyT. The authors thank Rodrigo Olea and Julio Oliva for interesting comments, and they specially thank Alan Garbarz and Mauricio Leston for collaboration and discussions. G.G. is grateful to the members of Pontificia Universidad Católica de Valparaíso for the hospitality during his stay; specially to Olivera Mišković.
[1] D. Anninos, W. Li, M. Padi, W. Song, A. Strominger, JHEP 0903 (2009) 130.

[2] G. Compère, S. Detournay, Class. Quant. Grav. 26 (2009) 012001; Erratum-ibid. 26 (2009) 139801.

[3] G. Compère, S. Detournay, JHEP 0908 (2009) 092.
[4] M. Henneaux, C. Martínez, R. Troncoso, Phys. Rev. D84 (2011) 124016 .

[5] M. Guica, arXiv:1111.6978.

[6] D. Anninos, M. Esole, M. Guica, JHEP 0910 (2009) 083.

[7] D. Anninos, JHEP 1002 (2010) 046. 
[8] M. Blagojević, B. Cvetković, JHEP 0905 (2009) 073.

[9] M. Blagojević, B. Cvetković, JHEP 0909 (2009) 006.

[10] O. Mišković, R. Olea, JHEP 0912 (2009) 046.

[11] S. Detournay, T. Hartman, D. Hofman, arXiv:1210.0539.

[12] K. Balasubramanian, J. McGreevy, Phys. Rev. Lett. 101 (2008) 061601.

[13] D.T. Son, Phys. Rev. D78 (2008) 046003.

[14] I. Bengtsson, P. Sandin, Class. Quant. Grav. 23 (2006) 971.

[15] M. Guica, T. Hartman, W. Song, A. Strominger, Phys. Rev. D80 (2009) 124008.

[16] G. Compère, S. Detournay, JHEP 0703 (2007) 098.

[17] W. Song, A. Strominger, JHEP 1205 (2012) 120.

[18] S. Detournay, Phys. Rev. Lett. 109 (2012) 031101.

[19] S. Detournay, D. Israel, J. M. Lapan, M. Romo, JHEP 1101 (2011) 030.

[20] T. Azeyanagi, D. M. Hofman, W. Song, A. Strominger, arXiv:1207.5050.

[21] M. Gary, D. Grumiller, R. Rashkov, JHEP 1203 (2012) 022.

[22] A. Bouchareb, G. Clément, Class. Quant. Grav. 24 (2007) 5581.

[23] K. Amit Moussa, G. Clément, C. Leygnac, Class. Quant. Grav. 20 (2003) L277.

[24] G. Clément, Class. Quant. Grav. 26 (2009) 105015.

[25] J. Brown, J. York, Phys. Rev. D47 (1993) 1407.
[26] G. Giribet, M. Leston, JHEP 1009 (2010) 070.

[27] E. Tonni, JHEP 1008 (2010) 070.

[28] S. Nam, J-D. Park, S-H. Yi, Phys. Rev. D82 (2010) 124049.

[29] E. Bergshoeff, O. Hohm, P. Townsend, Phys. Rev. Lett. 102 (2009) 201301.

[30] M. Bañados, C. Teitelboim, J. Zanelli, Phys. Rev. Lett. 69 (1992) 1849.

[31] R. Wald, Phys. Rev. D48 (1993) 3427.

[32] O. Hohm, E. Tonni, JHEP 1004 (2010) 093.

[33] C. Fefferman, R. Graham, Soc. Math. de France, Astérisque, hors série 95 (1985).

[34] J. D. Brown, M. Henneaux, Commun. Math. Phys. 104 (1986) 207.

[35] J. Oliva, D. Tempo, R. Troncoso, JHEP 0907 (2009) 011.

[36] E. Ayón-Beato, A. Garbarz, G. Giribet, M. Hassaïne, Phys. Rev. D80 (2009) 104029.

[37] V. Balasubramanian, P. Kraus, Commun. Math. Phys. 208 (1999) 413.

[38] S. Deser, R. Jackiw, S. Tempelton, Annals Phys. 140 (1982) 372; Phys. Rev. Lett. 48 (1982) 975.

[39] L.F. Abbott, S. Deser, Nucl. Phys. B195 (1982) 76.

[40] S. Deser, B. Tekin, Phys. Rev. Lett. 89 (2002) 101101; Phys. Rev. D67 (2003) 084009. 